\title{
LARGE SCALE ROAD NETWORK EXTRACTION IN FORESTED MOUTAINOUS AREAS USING AIRBORNE LASER SCANNING DATA
}

\author{
António Ferraz ${ }^{1,2}$, Clément Mallet ${ }^{1}$, Nesrine Chehata ${ }^{3,4}$ \\ 1. IGN/MATIS lab., Université Paris Est Marne la Vallée, France \\ 2. 2- R\&D Unit INESC Coimbra, R. Antero de Quental 199, 3000-033 Coimbra, Portugal \\ 3. Laboratoire G\&E (EA 4592), IPB / Université de Bordeaux, France \\ 4. IRD/UMR LISAH El Menzah 4, Tunis, Tunisia
}

\begin{abstract}
In this paper, we present an approach that is able to deal with large-scale road network extraction in montaneous forested areas. While former methods focus on delineating patches of roads without computing a coherent road network, we formulate a very large number of road hypothesis that are pruned using a graph reasoning and weak a priori knowledge on road behavior. The initial solution is computed by means of two machine learning and pattern recognition state-of-the-art methods (namely, Random Forest classification and Marked Point Process) that allow to process very large areas in little time with very satisfactory results. The approach was tested over a large area $\left(1400 \mathrm{~km}^{2}\right)$ providing satisfactory results.
\end{abstract}

Index Terms - Road network extraction, airborne laser scanning data, large scale, forest, mountainous areas.

\section{INTRODUCTION}

Knowledge on the forest mountain roads network is essential for many activities such as forest management, wood harvesting industry, wildfire protection and fighting. In France, the few national databases available, even those created by manual plotting and GPS-based digitization, are no longer up-todate due to both acquisition time and cost issues (especially in large area mountainous environments where the topography of the terrain is rather complex) of the field-based techniques currently used to map forest roads. As a result, the scientific community has been evaluating the reliability of remote sensing data to perform such a task. A large body of literature has tackled the problem in the last twenty years [1] but mainly focused on urban and peri-urban areas [2] where the object "road" is easy to model (constant width, perpendicular and parallel to other roads etc). Indeed, in forested environments, geospatial optical images are not adapted for forest road mapping because roads are totally or partially occluded by vegetation $[3,4]$ which even prevents gap-closure methods to be efficient [5]. On the contrary, airborne topographic laser scanning (ALS) is able to penetrate the vegetation and can measure the ground beneath. Therefore, it provides timely and accurate Digital Elevation Models (DEM) over large areas. Former works proved that such DEM can reveal road patches located beneath highly dense forest canopy $[6,7,8]$. Existing approaches dedicated to map forest road networks using ALS data mainly rely on a two-stage approach.

1. First, road segments (or edge road segments) are identified using point/pixel-based classification with standard image processing and machine learning methods [7, 9]. The road is modelled as locally planar, that is why slope and roughness-based features, computed on the Digital Terrain Model (DTM), are often employed.

2. Since only unconnected road segments are retrieved, the second step attempts to bridge gaps due to vegetation occlusion in order to derive a full road network [10]. So far, no automatic and robust method has been proposed to grow and link such segments in forested areas over large areas. This is in particular due to the fact that no strong prior knowledge can be applied in such unconstrained natural environments.

Similarly to [9], in this paper, we adopt a coarse-to-fine method which consists in first retrieving the most obvious road patches and then refining and completing their location. However, conversely to the latter approach, both local and global analysis are merged into a single framework so as to benefit from a multi-scale approach and alleviate the standard vegetation occlusion issue. In addition, we present an approach that is able to deal with large-scale road network mapping whereas existing approaches have only been applied to areas with limited spatial extension.

\section{METHOD}

The proposed workflow is composed of 5 steps and is fully based on the analysis of the terrain. We model a road as a local planar area, that is elongated in a specific direction, and that exhibits significant slope variation at its borders.

First, a filtering step separates ground and off-ground ALS 
3D points in order to compute a high-resolution Digital Terrain Model (DTM, $1 \times 1 \mathrm{~m})$ using a the standard TIN-based method [11]). The DTM spatial resolution was selected in order to propose a suitable trade-off between tiny segment discrimination and computing times.

Then, three DTM-based feature images were computed so as to subsequently discriminate road segments: roughness, slope, and a multi-scale slope gradient. The two first attributes are standard in the literature whereas the last one aims to enhance areas that are planar in a small neighborhood ( $2 \mathrm{~m})$ but not in a larger one $(12 \mathrm{~m})$, as illustrated in Figure 1.

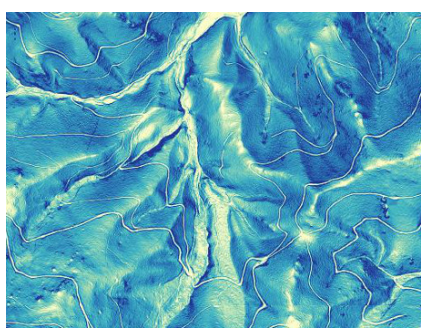

(a)

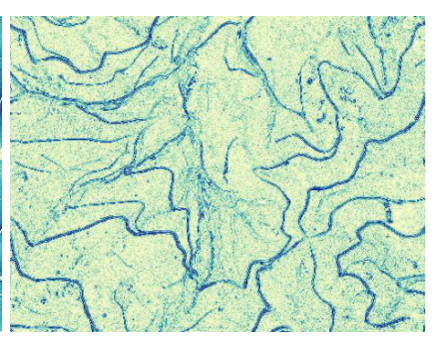

(b)
Fig. 1. Feature computation for classification. (a) DTMbased slope computation. (b) Multi-scale slope gradient retrieval. Yellow: low $\rightarrow$ Blue: high values.

In the third step, a Random Forest (RF) classifier [12] was fed with the referred feature images as well as with a training dataset corresponding to the two classes of interest: "road" and "non-road". The supervised RF technique is adopted since it exhibits two important features for our study: a high generalization ability and fast computing times. An improved version of the Sherwood library is used for that purpose [13]. The training samples correspond to less than 20,000 manually selected pixels. Such process is not rhedibitory for large scale mapping since it only represents $0.001 \%$ of the entire study site and the forest is composed of 100 trees. In addition to a binary decision (Figure 1a), the RF classifier provides a confidence map corresponding to the likelihood of a given pixel to be correctly classified (Figure 2b). Such confidence map is used to find road seeds that will be the basis of the road network. Consequently, the noisy results are pruned by applying an object-based filtering approach using mathematical morphology. Only pixels following a given direction are kept for graph generation: the process is applied every $25 \varnothing$. Then, graph vertices come from a slight downsampling of the remaining pixel set of highest confidence. For that purpose, a stochastic framework is adopted by means of a Marked Point Process, [14]. The optimal spatial seed point distribution is computed using an energy minimization framework according to two criteria: seed points located on pixels classified as a "road" with high confidence are highly favored, and seed points closer than 10 meters to each other are strongly penalized. For that purpose, the open-source library librjmcmc is adopted [15]. One can see on Figure $2 \mathrm{c}$ that most of the seed points are located on the road network. It allows to highly reduce the computational effort for the subsequent tasks since for $1 \mathrm{~km}^{2}$ only $\sim 1000$ seeds are now taken into account instead of $1 \mathrm{M}$ pixels. In addition, the Marked Point Process was adopted since an efficient implementation was available: the optimal solution is found after $17 \mathrm{M}$ iterations, which corresponds to $14 \mathrm{~s}$.

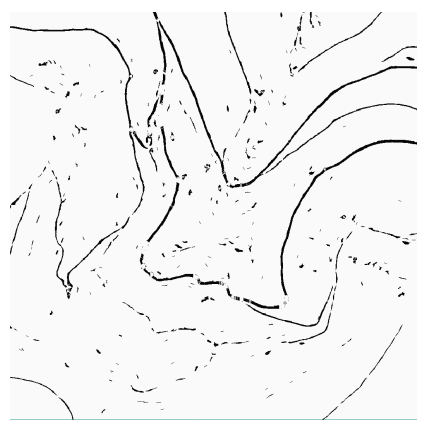

(a)

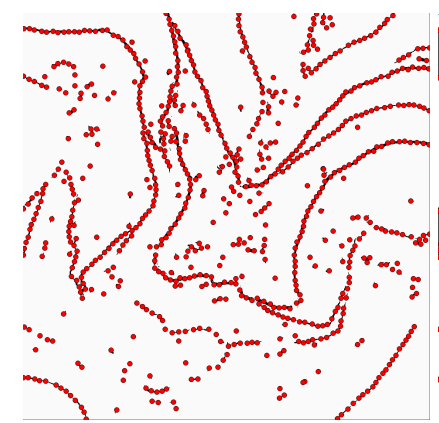

(c)

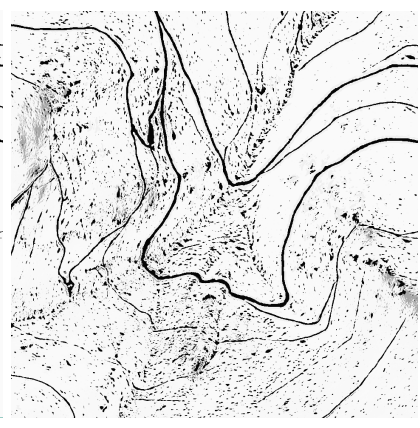

(b)
Fig. 2. Graph establishment. (a) binary image displaying the classes "road" (in black) and "non-road" (in white). (b) confidence image according to the RF classifier (black, high confidence; gray, moderate confidence and white, low confidence). (c) spatial distribution of the graph vertex according to the marked point process. (d) graph edge computation.

Then, a graph is computed by connecting each seed point with its closest neighbors on the 8 main cardinal point directions (Figure 2d). This allows creating connections between road patches even in presence of significant occlusions or missing data (no 3D ALS points on the ground). Furthermore, this allows linking parallel roads where significant slope exist and that cannot be distinguished with the RF classification. In the last step, the graph is pruned in order to remove irrelevant edges: a weight is assigned to each edge so as to evaluate whether a given edge represents a road segment (Figure $3 \mathrm{a}$ ). Briefly speaking, lower weights are assigned to edges that cross pixels with similar values in terms of both slope and roughness (derived from the DTM computed in the first step). Therefore, as lower weight edges are likely to be a road segment, edges with weights lower than a given threshold are 
kept and removed otherwise (Figure 3a). Such threshold is computed as a function of the training dataset introduced by the end-user for the supervised classification step. Finally, the whole process leads to a vector map that corresponds to the forest road network of the study site (Figure 4).

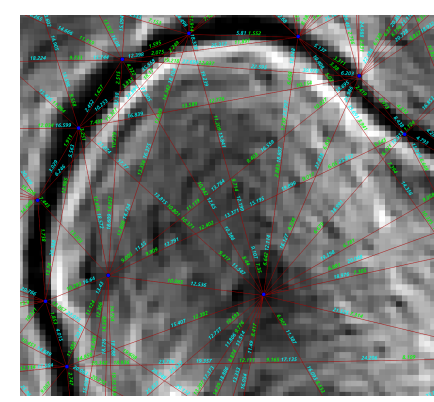

(a)

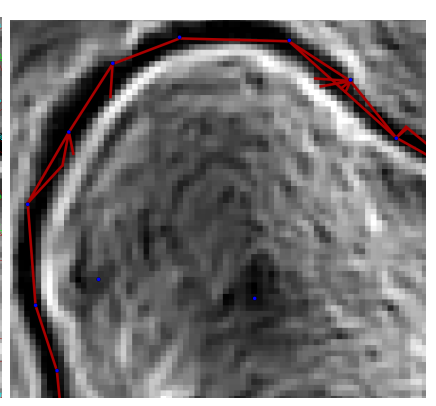

(b)
Fig. 3. Image of the terrain slope computed from the DEM: (a) weighted graph edges, and (b) graph after pruning.

\section{RESULTS}

The reliability of the method was assessed on a study area that covers approximately $1425 \mathrm{~km}^{2}$ of the Vosges Mountains located in Eastern France with an altitude ranging from $200 \mathrm{~m}$ to $1364 \mathrm{~m}$. The forest cover is predominantly dominated by European spruce (Picea abies L.), Scots pines (Pinus sylvestris L.), Sycamore maple (Acer pseudoplatanus L.), European beech (Fagus sylvatica L.) and Sessile oak (Quercus petrea Liebl.). The study site measurements were carried out using an ALS Optech 3100EA sensor using a scan angle of \pm $17^{\circ}$ with a footprint of $0.8 \mathrm{~m}$. The ALS point density ranges from 2 to $4 \mathrm{pt} / \mathrm{m}^{2}$ which fits with operational constraints of nation-wide surveys.

The method was visually compared to two forest road databases maintained by the French National Institute of Geographic Information and Forestry (IGN, Institut National de l'Information Gographique et Forestire) and the National Forest Office (ONF, Office National des Forêts, Figure 4). Both of them have been created with a combination of orthoimage-based manual digitizing and field surveys using GPS. Consequently, they are likely to provide a complete road netwok but without very high planimetric accuracy, which prevents a straightforward comparison between our method and these two geospatial databases. The result indicate that our method succeeds in areas with complex topography: many roads that are not recorded in the current databases due to dense vegetation cover or complex topography are correctly extracted (see Figure 5). In addition, even when roads already exist, their planimetric location is better retrieved with our method, suggesting that it can be used for updating databases. Moreover, two main limitations can be noticed. First, the approach fails in environments with low relief and urban areas since they violate our road model. Secondly, in the final edge pruning step, the constructed network can be broken, resulting in the loss of a full road network.

Finally, the computing times show that our method is tailored to reliably process large areas in a suitable time: it took 58 hours for $1400 \mathrm{~km}^{2}$ for a single-core computer i.e., 2'25" per $\mathrm{km}^{2}$.

\section{CONCLUSION}

The proposed method was able to correctly map forest roads in mountainous areas and, in addition, can be used to update existing databases. Using a graph as an initial solution allowed to exploit the continuous homogeneous nature of a road and to derive a final solution that meets the requirements in terms of a road network, i.e., it generates few unconnected road segments out of the main network. The method was tailored to be fast, using efficient implementation of machine learning and pattern recognition tools, and with the simple knowledge on the Digital Terrain Model. However, in order to refine the current extraction and improve both classification and graph generation, it is now necessary to adopt more complex and top-down approaches [16, 17].

\section{ACKNOWLEDGEMENTS}

This work was supported by the French National Research Agency (ANR) through the FORESEE project (ANR-2010BIOE-008).

\section{REFERENCES}

[1] J.B. Mena, "State of the art on automatic road extraction for GIS update: a novel classification," Pattern Recognition Letters, vol. 24, no. 16, pp. 3037-3058, 2003.

[2] S. Clode, F. Rottensteiner, P. Kootsookos, and E. Zelniker, "Detection and vectorization of roads from lidar data," Photogrammetric Engineering \& Remote Sensing, vol. 73, no. 5, pp. 571-535, 2007.

[3] H. Jiuxiang, A. Razdan, J. Femiani, C. Ming, and P. Wonka, "Road network extraction and intersection detection from aerial images by tracking road footprints," IEEE Transactions on Geoscience and Remote Sensing, vol. 45, no. 12, pp. 4144-4157, 2007.

[4] A. Shackelford and C. Davis, "Fully automated road network extraction from high-resolution satellite multispectral imagery," in IEEE International Geoscience and Remote Sensing Symposium, 2003, pp. 461-463.

[5] M. Rochery, I. Jermyn, and J. Zerubia, "Gap closure in (road) networks using higher-order active contours," in IEEE International Conference on Image Processing, pp. 1879-1882.

[6] W. Rieger, M. Kerschner, T. Reiter, and F. Rottensteiner, "Roads and buildings from laser scanner data in a forest enterprise," International Archives of Photogrammetry and Remote Sensing, vol. 32 (Part 3/W14), pp. 185-191, 1999. 


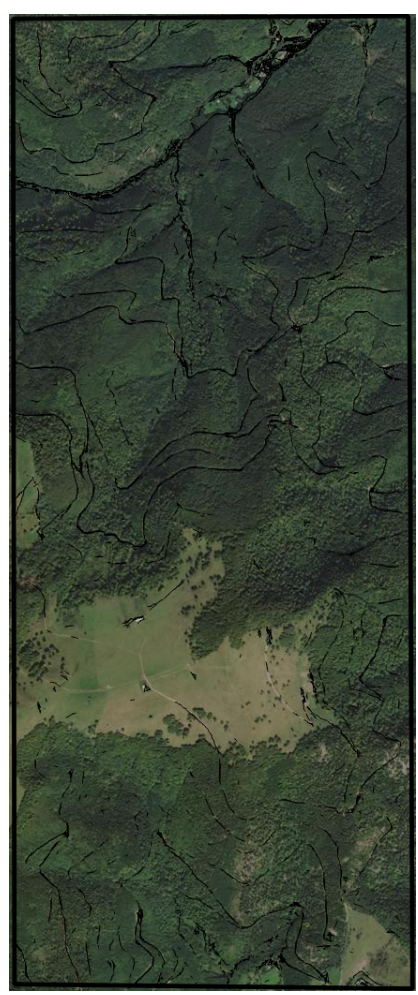

(a)

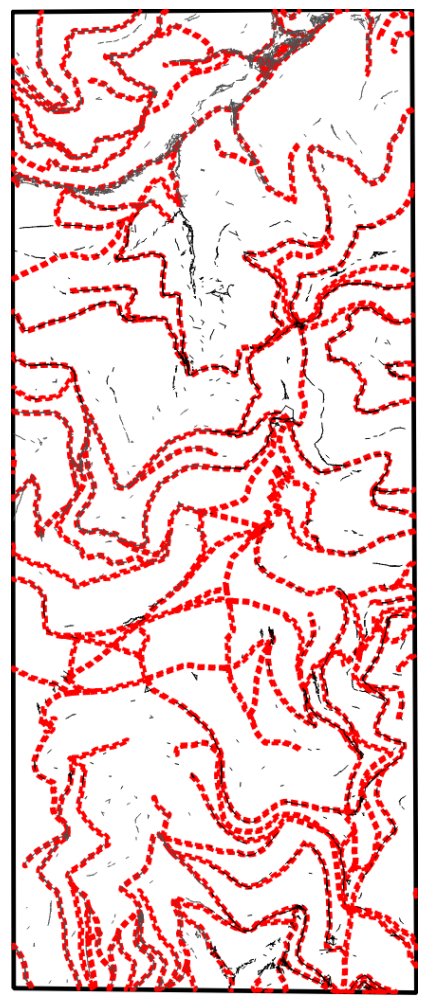

(c)

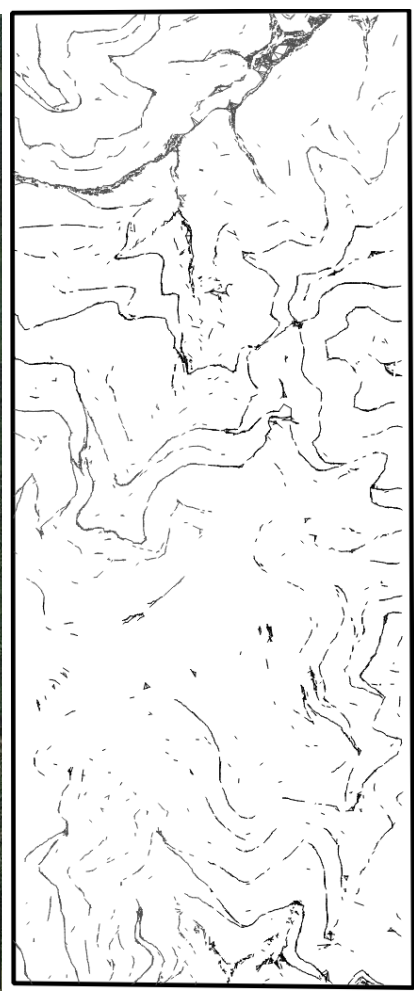

(b)

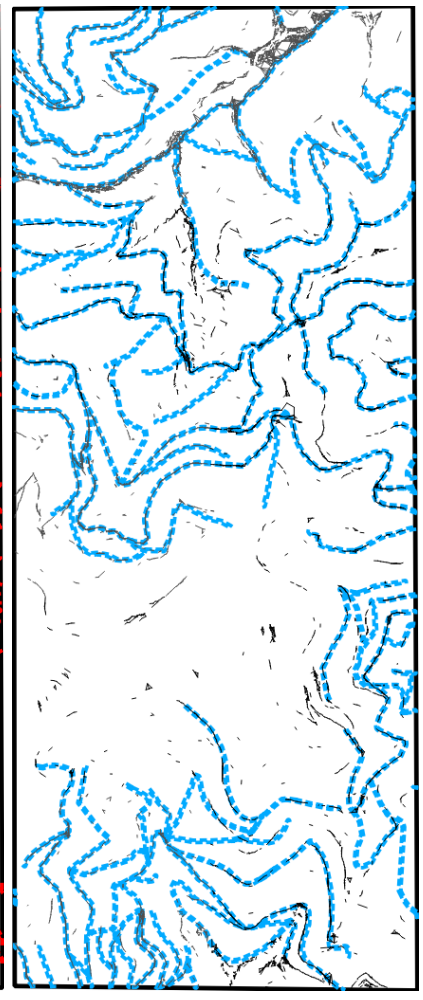

(d)
Fig. 4. Focus on a small patch of the area of interest $(5 \mathrm{~km} \times 2 \mathrm{~km})$. (a) Aerial orthoimage (C) IGN. (b) Result of our method (black). (c) ONF database (red). (d) IGN database (blue).

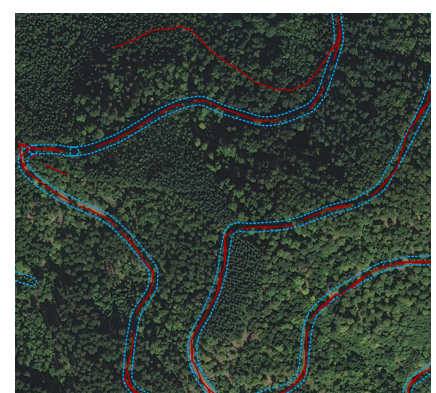

(a)

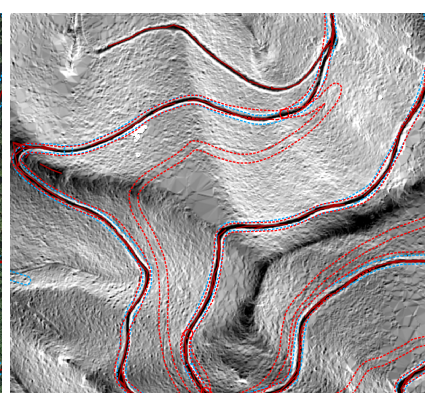

(b)
Fig. 5. Focus on dense vegetation area where our method performs better that existing topographic databases. (a) Our extraction (continuous red line) compared to the IGN database (dashed blue lines). (b) Same results with the ONF database (dashed red lines) superimposed on the ALS DTM.

[7] N. David, C. Mallet, T. Pons, A. Chauve, and F. Bretar, "Pathway detection and geometrical description from als data in forested mountaneous areas," International Archives of Photogrammetry, Remote Sensing and Spatial Information Sciences, vol. 38 (Part 3/W8), pp. 242247, 2009.

[8] R. White, B. Dietterick, T. Mastin, and R. Strohman, "Forest roads mapped using lidar in steep forested terrain," Remote Sensing, vol. 2, no. 4, pp. 1120-1141, 2010.

[9] A. Djuricic and M. Hollaus, "Extraction of forest roads from full-waveform airborne laser scanning data," in $E G U, 2013$.

[10] C. Hatger, "On the Use of Airborne Laser Scanning Data to Verify and Enrich Road Network Features," International Archives of Photogrammetry, Remote Sensing and Spatial Information Sciences, vol. 36 (Part 3/W19), pp. 138-143, 2005.

[11] A. Soininen, "http://www.terrasolid.fi," Website of the Terrasolid software packages, 2014, Accessed: April 30, 2014.

[12] L. Breiman, "Random forests," Machine Learning, vol. 45, no. 1, pp. 5-32, 2001.

[13] A. Criminisi and J Shotton, Eds., Decision Forests for Computer Vision and Medical Image Analysis, Springer, 2013.

[14] X. Descombes, Ed., Stochastic Geometry for Image Analysis, Wiley, 2011.

[15] M. Brédif and O. Tournaire, "LibRJMCMC: An opensource generic C++ library for stochastic optimization," ISPRS Archives, vol. XXXIX-B3, pp. 259-264, 2012.

[16] Dengfeng Chai, Wolfgang Forstner, and Florent Lafarge, "Recovering line-networks in images by junctionpoint processes," in IEEE conference on Computer Vision and Pattern Recognition, 2013.

[17] J.D. Wegner, J.A. Montoya-Zegarra, and K. Schindler, "A higher-order crf model for road network extraction," in IEEE Conference on Computer Vision and Pattern Recognition, 2013. 